This is an Open Access article, distributed under the terms of the Creative Commons Attribution licence (http://creativecommons.org/licenses/by/4.0/), which permits unrestricted re-use, distribution and reproduction, provided the original article is properly cited.

\title{
ERRATUM
}

\section{Direct construction of optimized stellarator shapes. Part 3. Omnigenity near the magnetic axis - ERRATUM}

\author{
Gabriel G. Plunk ${ }^{\circledR 1}$ †, Matt Landreman $^{\circledR 2}$ and Per Helander ${ }^{\circledR 1}$ \\ ${ }^{1}$ Max-Planck-Institut für Plasmaphysik, EURATOM Association, 17491 Greifswald, Germany \\ ${ }^{2}$ Institute for Research in Electronics and Applied Physics, University of Maryland, College Park, \\ MD 20742, USA
}

doi:10.1017/S002237781900062X, Published by Cambridge University Press, 5 November 2019

Below we clarify the conventions used for normalization in our paper (Punk, Landreman \& Herlander 2019), and correct some associated errors in the equations. The validity of the numerical solutions and the main conclusions of the paper are unaffected by these corrections.

Following Garren \& Boozer (1991), we define the expansion parameter as $\epsilon=\sqrt{\psi}$ so that the magnetic field can be expressed to first order as $B(\epsilon, \theta, \varphi) \approx B_{a}(\varphi)(1+$ $\left.\epsilon \sqrt{2 / B_{a}(\varphi)} \kappa^{s}(\varphi) \eta_{\mathrm{GB}}(\varphi) \cos [\theta-\alpha(\varphi)]\right)$, where the ' $\eta$ ' of Garren \& Boozer (1991) is here denoted $\eta_{\mathrm{GB}}$; see their (79). For simplicity, we introduced the quantity $d$, related to $\eta_{\mathrm{GB}}$ by

$$
d(\varphi)=\sqrt{\frac{2}{B_{a}(\varphi)}} \kappa^{s}(\varphi) \eta_{\mathrm{GB}}(\varphi),
$$

so that the magnetic field to first order becomes

$$
B(\epsilon, \theta, \varphi) \approx B_{a}(\varphi)(1+\epsilon d(\varphi) \cos [\theta-\alpha(\varphi)]),
$$

as correctly written in (6.1) of our paper.

Our definitions for $d$ and $\epsilon$ affect the forms of the first order components of the coordinate mapping, $X_{1}$ and $Y_{1}$. These quantities are introduced in the text at the beginning of $\S 7$, where the the coordinate mapping $x$ to first order should read

$$
\boldsymbol{x} \approx \boldsymbol{r}_{0}+\epsilon\left(X_{1} \boldsymbol{n}^{s}+Y_{1} \boldsymbol{t}^{s}\right) .
$$

Note the factor of $\epsilon$ is missing in the paper. The form of $X_{1}$ was correctly given by (7.1), but that for $Y_{1},(7.2)$, should read

$$
Y_{1}=\frac{2}{B_{a}(\varphi) \bar{d}(\varphi)}\{\sin [\theta-\alpha(\varphi)]+\sigma(\varphi) \cos [\theta-\alpha(\varphi)]\},
$$

$\dagger$ Email address for correspondence: gplunk@ipp.mpg.de 
with the correction being the factor of $1 / B_{a}(\varphi)$.

Our forms of $B, X_{1}$ and $Y_{1}$ can be compared with (79)-(81) of Garren \& Boozer (1991), and can be seen to agree, given our definitions of $\epsilon, d$ and $\bar{d}$, and the substitution $\kappa \rightarrow \kappa^{s}$. A related error was introduced into the definition of $P$ immediately following (7.7), which should read

$$
P=1+B_{a}^{2} \bar{d}^{4} / 4
$$

The solutions presented in $\S 8$ remain valid, but we note that $d$ defined in (8.4) (also depicted in figure $2 b$ ) misses a factor of $\sqrt{2}$, and should read

$$
d(\varphi)=\sqrt{2}[1.08 \sin (\varphi)+0.26 \sin (2 \varphi)+0.46 \sin (3 \varphi)] .
$$

Finally, there is a typo, unrelated to the preceding issues: following (7.10) it should read $\Delta \varphi(\varphi)=\varphi-\varphi_{b}(\varphi)$.

\section{Acknowledgements}

We wish to thank to R. Jorge and K. Camacho Mata for helping to discover and correct these errors.

Editor Peter Catto thanks the referees for their advice in evaluating this article.

\section{REFERENCES}

Garren, D. A. \& Boozer, A. H. 1991 Magnetic field strength of toroidal plasma equilibria. Phys. Fluids B 3 (10), 2805-2821. Available at: http://scitation.aip.org/content/aip/journal/pofb/3/10/10. 1063/1.859915.

Punk, G. G., Landreman, M. \& Herlander, P. 2019 Direct construction of optimized stellarator shapes. Part 3. Omnigenity near the magnetic axis. J. Plasma Phys. 85 (6), 905850602. 